\title{
Stress in Post-Secondary: Toward an Understanding of Test- Anxiety, Cognitive Performance, and Brief Mindfulness Meditation
}

\author{
Raychel Colangelo ${ }^{1} \&$ Karyn Audet ${ }^{2}$
}

\author{
Review Editors \\ Gop Majak ${ }^{3}$, Kate Briand ${ }^{4}$, Alain Morin ${ }^{5}$, Brant Downey ${ }^{6}$ \\ Editor \\ Shayla-Rose Somers ${ }^{7}$
}

\begin{abstract}
Premised on cultivating present-moment awareness, mindfulness meditation (MM) programs have been shown to significantly reduce state-anxiety and improve cognitive performance in post-secondary students. With increasing popularity, briefer MM formats have been introduced to post-secondary institutions to combat the rising prevalence of student test-anxiety. However, research examining the efficacy of brief MM on a state-level test-anxiety response and its ability to improve cognitive performance in a testing situation is sparse. The present study examined the immediate effects of brief MM on state test-anxiety and cognitive performance. A sample of 50 undergraduate college students $(N$ $=50$ ) were randomly assigned to a brief MM or a control activity. In the current study, it was hypothesized that state test-anxiety levels would be lower in the brief MM group than the control group and that those in the brief MM group would score higher on a cognitive performance test than the control group. Results revealed that the brief MM group had greater state test-anxiety reduction than the control group at post-treatment. Consistent with previous work, brief $\mathrm{MM}$, however, did not promote any specific short-term benefits for cognitive performance. Our findings, however, converge with past research to suggest that brief MM may produce immediate, short-term state test-anxiety relief. Immediate anxiety relief may be beneficial for students during stressful academic periods.
\end{abstract}

Keywords: state-anxiety, test-anxiety, cognitive performance, mindfulness, college students

\footnotetext{
1 BA Psychology (Honours) · Douglas College · raychel.colangelo@gmail.com

$2 \mathrm{PhD} \cdot$ Department of Psychology $\cdot$ Douglas College

3 BA Psychology Student · Mount Royal University

4 BA Psychology (Honours) · Mount Royal University

$5 \mathrm{PhD} \cdot$ Department of Psychology $\cdot$ Mount Royal University

$6 \mathrm{PhD} \cdot$ Department of Sociology $\cdot$ Mount Royal University

7 BA Psychology Student (Minors: Women \& Gender Studies; Biology) • Mount Royal University
} 


\section{Stress in Post-Secondary}

Tests are ubiquitous in students' academic experiences, with most post-secondary institutions necessitating testing of questionable value (Brady et al., 2018). The intensifying pressure to perform well on tests can result in experiences of testanxiety, a distressing emotional state characterized by cognitive, behavioral, and physiological responses elicited by a testing situation (Spielberger, 1972; Zeidner, 1998). A study of 1,099 Canadian university students indicated that 38.5 percent (46.3 percent of females and 30.0 percent males) reported experiencing test-anxiety at one point in their academic careers (Gerwing et al., 2015).

Incidence of test-anxiety in college age populations have been estimated to range between 15-40 percent (Abolghasemi et al., 2009; Gerwing et al., 2015; von der Embse et al., 2013), with females reporting higher levels of test-anxiety than males (Nausheen et al., 2019). Previous work suggests that test-anxiety can significantly impair cognitive performance, namely test scores (Chin et al., 2017). As such, a more thorough examination of test-anxiety is necessary on campus settings.

\section{Trait-State Distinction of Anxiety}

As testing continues to increase, a dramatic rise is test-anxiety has been observed in college students (Gerwing et al., 2015). Test-anxiety can be conceptualized in the context of a trait-state distinction. State-test anxiety, in particular, is a current emotional state characterized by feelings of tension, worry, and apprehension induced by a testing situation (Spielberger, 1966). Comprised of several sub-elements, state test-anxiety can be exasperated by the level of difficulty of questions, ability and knowledge of test subject matter, prior exam preparation, and individual differences in anxiety susceptibility (trait-anxiety) (Spielberger \& Vopp, 1995).

As a result, the cognitive processes required to perform well on tests can be impacted, thereby leading to an increase in mind-wandering (Beilock, 2011; see also Chin et al., 2017; Zeidan et al., 2010a; Xu et al., 2017). Increased worry responses, greater frequency of mind-wandering, and decreased focus during exams can result in learning and memory disruptions, poor test performance, low selfefficacy, and hampered academic achievement (Chin et al., 2017; see also Mrazek et al., 2013; Sapp,
2014; Spielberger et al., 1976; Stoker \& Perkin, 2014; Thomas et al., 2017).

Given the effects of state test-anxiety on students, suitable stress-management options are necessary within college campus support services.

\section{Mindfulness Meditation in Post-Secondary Education}

Academic institutions have explored various stress-management options including exercise and wellness programs, counselling services, animalassisted interventions, and mindfulness training (Dvořáková et al., 2017; see also Galante et al., 2018; Jayawardene et al., 2017; Trammell, 2017).

In the current study, one such approach that will be examined in depth is mindfulness meditation (MM). Rooted in Buddhist tradition, MM refers to a mental training practice that involves the cultivation of nonjudgmental, moment-to-moment awareness. MM aims to refocus one's attention on their internal and external experiences without judgement by emphasizing the following key components: 1) focused attention (limiting attention to one particular object) and 2) detached observation (the process of expanding one's attention from one object to experience all physical and mental events through a series of inbreaths and outbreaths) (Kabat-Zinn, 1982; Kabat-Zinn, 2003).

As thoughts, emotions, and sensations arise, meditators are encouraged to bring their attention to their breath, refocus their attention to the present-moment, note their physical and/or mental states, and experience them as they are (Kabat-Zinn, 1982). Delivered online or in-person, MM programs have typically ranged between eight to ten weeks. The most popular MM psychotherapy programs include Mindfulness-Based Stress Reduction (MBSR) and Mindfulness-Based Cognitive Therapy (MBCT).

Typically delivered in a weekly group psychotherapy format, these rigorous mental training programs consist of countless hours of mindfulness practice, including a one-day mindfulness retreat, weekly group meetings, intensive at-home assignments, and lengthy meditation and yoga instruction (Kabat-Zinn, 1982). Despite showing evidence of efficacy, these intensive programs may be especially challenging for students who already face various academic pressures, time constraints, and extracurricular activities. 
Briefer formats of MM may be more suitable for post-secondary students. Recent research has examined simplified versions of MM programs, such as one-session, one-week, and two-week sessions (Banks et al., 2015; see also Cavanagh et al., 2013; Wells \& Roussis, 2014). Variance among the duration of previous brief MM studies may be attributed to the ambiguity surrounding the necessary minimal amount of time needed to achieve mindfulness. The present study aimed to add to the literature concerning the efficacy a single-session MM on test-anxiety. Brief MM may be particularly beneficial for students faced with various academic pressures, such as increased workloads and lack of financial freedom (Santorelli, 2014).

Brief MM has been evidenced to effectively improve psychological well-being in nonclinical college student populations. Broderick (2005) found that an eight-minute MM session significantly reduced negative affectivity and dysphoric mood in a college sample when compared to controls. Similarly, a recent study by Johnson et al. (2015) found that a 25-minute session of MM positively enhanced mood states when compared to controls.

In another undergraduate sample, Zeidan et al. (2010b) a three-session study comparing the effects of MM to unguided meditation session (guided instructions is central to $\mathrm{MM}$ ) and no intervention on mood found that 20-minutes of $\mathrm{MM}$ a day reduced state-anxiety scores more effectively than controls at post-treatment. Similarly, Ainsworth et al. (2015) found that a 10minute MM session has also been shown to reduce state-anxiety and enhance positive mood at posttreatment when compared to brief relaxation training.

Brief MM has also been shown to enhance psychological mechanisms that improve cognitive performance, such as working memory and executive attention (Morrison et al., 2014; see also Taren et al., 2017; Zeidan et al., 2010a; Zeidan et al., 2009). In Taren et al.'s (2017) three-day study, the fMRI scans of those who participated in brief MM had greater executive control, attention, working memory, and cognitive performance than those who participated in an intensive relaxation training program.

Congruently, Zeidan et al. (2010a) found that four, 20-minute MM sessions significantly reduced state-anxiety scores and enhanced meditators' abilities to sustain attention, inhibit mindwandering, and reduce distraction when compared to controls. Similarly, Zeidan at al. (2009) found that a brief three-day MM reduced self-reported pain and anxiety ratings by sustaining attention and enhancing ability to focus on the present moment in comparison to relaxation training or cognitive distractor tasks. Improved cognitive performance may be particularly beneficial for students during a stressful testing situation.

Research examining the effectiveness of brief MM on state test-anxiety is sparse, however available evidence indicates that mindfulnessbased interventions may reduce test-anxiety and improve cognitive performance by cultivating emotional regulatory skills, enhancing working memory and inhibiting task-irrelevant thoughts (Calma-Birling \& Gurung, 2017; Mrazek et al., 2013).

For instance, six days of self-administered mindful breathing practices were found to reduce test-anxiety scores through the mitigation of automatic negative thoughts and the reappraisal of worry cognitions (Cho et al., 2016). These results further converge with those of Lothes II and Mochrie (2017) who found that an eight-week Dialectical Behavior Therapy (DBT) mindfulness program significantly reduced self-reported testanxiety and general anxiety in a college student sample at post-treatment.

Brief MM has also been evidenced to improve cognitive performance in college student populations. Mrazek et al. (2013), for instance, investigated the effects of a two-week MM program and its ability to improve performance on the General Records Exam (GRE). Researchers found that brief MM not only reduced interfering thoughts, inhibited mind-wandering, and improved working memory during the completion of the GRE, it showed improvement in GRE test scores when compared to a control (Mrazek et al., 2013).

Improved testing performance was attributed to an increase in mindfulness and a decrease in distracting thoughts among test-takers in the brief MM group brief (Mrazek et al., 2013). Similarly, in Dundas et al.'s (2016) longitudinal study, five brief MM sessions held over the course of two-weeks of a semester significantly reduced the cognitive and emotional components of test-anxiety exhibited among students. 


\section{Present Study}

In the present study, we sought to explore the gap in psychological research concerning the traitstate distinction of test-anxiety and investigate the immediate effects of a single 20-minute MM session on state- level test-anxiety in a college student sample. In addition, this study examines whether anxiety reduction via brief MM improves cognitive performance when compared to a control group. Based on past research, the hypotheses for the current study were that state test-anxiety levels will be lower in the brief MM group than the control at post-treatment, and that those who engage in brief MM will score higher on a cognitive performance test than those in the control.

\section{Method}

\section{Participants}

A power analysis based on aggregated means and standard deviations for a primary outcome measure (State Anxiety Inventory; Spielberger et al., 1983) at an alpha level of .01, power of .80 and an effect size of .25 indicated that 20 participants per group was needed to detect a significant difference. A convenience sample of 50 undergraduate college students $(N=50)$ volunteered in exchange for bonus research participation credit $\left(M_{a g e}=23.52, S D_{a g e}=7.56\right.$, Range= 18-46).

Participants were excluded if they were a) currently experiencing and/or had a history of extreme forms of anxiety (i.e., recurring panic attacks and/or excessive anxiety for more than 6 months), b) younger than 18 years old, and/or, c) enrolled in third or fourth year studies. Outlier criteria was set prior to the analysis $( \pm 4$ SDs from sample means) and two participants $(n=2)$ were identified and removed from all analyses. Analyses are reported on 48 participants (24 in each group), with 34 females and 14 males. All study procedures were approved by the College Institutional Research Ethics Board.

\section{Design}

This study employed a 2 X 2 mixed-model experimental design to test the effectiveness of brief MM on state- test anxiety levels and cognitive performance. Intervention (brief $\mathrm{MM}$ versus control) was the between-subjects factor and time (pre-and post-treatment) was the within-subjects factor.

\section{Measures}

\section{State Test-Anxiety Induction}

A 10-item test comprising of six multiplechoice items and four short-answer questions was used to induce a state test-anxiety. This Anxiety Induction Test (AIT) included questions that were selected by researchers based on personal choice from a pre-existing measure (The Impossible Quiz, 2018) and were determined by researchers and mock participants in a trial run to be difficult in nature. No scores were tallied on this test, and participants were told that this test was predictive of their intelligence and college success. The test featured items such as, "What is the 9-letter common word in English that will continue to be a real, commonly used English word after you take away one letter at a time until there is only one left (?)".

Participants were given five minutes to complete the AIT while a digital clock counted down. The digital clock was visible to participants and made a loud ticking noise that was intending to further increase anxiety.

\section{State-Anxiety Inventory}

The State-Anxiety Inventory (SAI) is a 20-item test used to measure state-level test-anxiety (Spielberger et al., 1983). The questionnaire requires participants to indicate how they feel "right now, at this moment" on items such as "I feel at ease" and "I feel upset" (Spielberger et al., 1983). Summing across questions rated on a four-point Likert scale (1=-not at all; $4=$ very much so), scores on this test have typically range from 20 to 80 with higher scores indicating high state-anxiety. The SAI has been widely used in psychological research to assess state-anxiety and demonstrates high testretest reliability ( $\alpha=.65-.75$, respectively) and internal consistency (ranging from $\alpha=.86-.95$, respectively) (Spielberger et al., 1983).

\section{Cognitive Performance}

A 20-item Cognitive Performance Test (CPT) was used to compare cognitive performance between groups. The test was designed from the 75-item Otis General Intelligence Examination (Otis, 1920) and was intended to be significantly easier than the AIT. An adaptation from the original 75-item test was necessary in order to accommodate for time restraints placed on data collection (i.e., duration of lab bookings). 
Items were arbitrarily selected by the researcher based on personal choice to include items of varying difficulty (easy to hard), assessed by mock participants in a trial run, and were then scored by the researcher using a predetermined response set supplied by the Otis General Intelligence Examination, with scores ranging from 0 to 20 . The adapted test featured 10 multiplechoice and 10 short-answer questions such as "The opposite of early is (?)" and "A boy's father's brother is his (?)". Participants were given 15 minutes to complete the test.

\section{Brief Mindfulness Meditation Intervention}

Participants in the brief MM group listened to a 20-minute MM audio recording guided by KabatZinn (2014), a world-renowned mindfulness facilitator and researcher. Participants were instructed to make themselves comfortable, close their eyes, and listen to the audio recording, without conversing with other participants. Through a series of inbreaths and outbreaths, in conjunction with silent practice periods and intermittent prompts during these intervals, the intervention instructed participants to notice their bodily sensations, attend to their physical and mental sensations, and nonjudgmentally experience them as they are.

A researcher in the front corner of the room monitored participants' attentiveness during the session. Participants kept their eyes closed and remained silent throughout the entire session. Temperature, lighting (dimly lit and window shades closed), and time of day remained constant between the brief MM group and the control group.

\section{Procedure}

Participants arrived at a college laboratory on one of two scheduled times between 8:30 am and 1:30 pm. Participants were recruited under the impression that the study intended to decrease stress and improve cognitive performance. To minimize experimenter expectancy effects, a written protocol was used throughout the study and did not differ between the two experimental sessions. In the laboratory, informed consent was obtained, and all participants went through the anxiety induction phase. Participants were told that they would be completing a test that was predictive of their intelligence and college success.

To further induce state-test anxiety, a large digital timer set at five-minutes was displayed and visible at the front of the room and loudly counted down as participants completed the AnxietyInduction Test. Participants then completed a demographics questionnaire (i.e., age, sex) and a baseline SAI measure. Participants were then randomly assigned (using a computerized random number-program) to one of two groups: brief MM $(n=24)$ or control $(n=24)$.

Participants in the brief MM group were asked to follow an assigned researcher to a separate (but similar) classroom, and to remain silent and refrain from speaking to anyone while moving to the other room. Participants in the brief MM group were then instructed to close their eyes and listen silently to a 20-minute MM audio recording while a researcher monitored at the front of the room.

Control participants were directed to remain in the original laboratory and were given a single piece of paper. Control participants were then instructed to create an origami piece by following a 20-minute instructional video tutorial (How to Origami, 2014). A researcher monitored attentiveness and reported no communication or interaction with other participants during the 20minute interval. Both activities were held in similar classrooms with similar lighting, temperature, and during the same time of day.

All participants then regrouped in the original laboratory and completed the SAI a second time, followed by the Cognitive Performance Test. At the end of both sessions, participants were debriefed, informed of the use of deception in the study, and were compensated with bonus credit.

\section{Results}

It was hypothesized that state test-anxiety levels would be lower in the brief MM group than the control group. It was also hypothesized that those in the brief MM group would score higher on the CPT than those in the control group. Mean SAI scores for all participants were obtained pre- and post-treatment. Mean cognitive performance scores were obtained at post-treatment. Difference scores were used in analyses involving SAI means.

Two separate independent samples t-tests were used to compare groups on SAI difference scores and CPT scores. The analyses were accomplished using the Statistical Package for Social Sciences (SPSS). An alpha level of. 05 was used for all statistical tests.

Preliminary analyses revealed no significant differences in gender on baseline SAI scores, such 
that females $(M=43.92, S D=11.67)$ and males $(M$ $=45.88, S D=12.09$ ) had similar state-anxiety levels at pre-treatment. Although not statistically significant, females $(M=-16.44, S D=13.96)$ had greater state-anxiety reduction than males $(M=$ 9.83, $S D=9.98$ ) at post-treatment. Table 1 compares the groups on demographic variables (e.g., age, sex) and baseline measures and shows that there were no significant differences.

SAI scores, presented in Table 2, were analyzed with an independent samples t- test using difference scores. Results indicated that the brief

\section{Table 1}

Mean Scores and Standard Deviations Baseline Measures of Each Group

\begin{tabular}{|c|c|c|c|c|c|c|c|c|}
\hline & Brief MM & $S D$ & $n$ & Control & $S D$ & $n$ & $t^{*} / \chi^{2}$ & $p$ \\
\hline Age & 23.92 & 7.51 & 24 & 22.67 & 7.28 & 24 & .585 & .561 \\
\hline Sex & & & & & & & .403 & .525 \\
\hline Male & $25.0 \%$ & & 6 & $33.3 \%$ & & 8 & & \\
\hline Female & $75.0 \%$ & & 18 & $66.7 \%$ & & 16 & & \\
\hline Prior & & & & & & & .356 & .551 \\
\hline \multicolumn{9}{|l|}{ Meditation } \\
\hline Yes & $41.7 \%$ & & 10 & $44.4 \%$ & & 8 & & \\
\hline No & $58.3 \%$ & & 14 & $66.7 \%$ & & 16 & & \\
\hline SAI & 45.08 & 14.33 & 24 & 45.50 & 9.11 & 24 & -.132 & .895 \\
\hline
\end{tabular}

Note. $\mathrm{MM}=$ mindfulness meditation; $S D=$ standard deviation; $n=$ number of participants in sample; $t / \chi^{2}=$ effect size for Chi-square test; $p=p$-value; SAI = State Anxiety Inventory.

${ }^{*} t=$ degrees of freedom $(\mathrm{df})$ of $\mathrm{t}$-distribution $=46$

MM group $(M=-14.79, S D=13.20)$ had greater state-anxiety reduction than the control group $(M$ $=-2.41, S D=12.71), t(46)=-3.30, p=.002, d=0.95$, such that reductions in state test-anxiety levels were significantly larger in the brief MM group than the control group at post-treatment. However, differences in cognitive performance scores for the brief MM $(M=11.13, S D=3.10)$ and control $(M$ $=11.70, S D=3.30$ ) group were not significant, $t(45)$ $=-.617, p=.540, d=0.22$.

Table 2

Mean Scores and Standard Deviations for Dependent Variables of Each Group

\begin{tabular}{|c|c|c|c|c|c|c|c|c|c|}
\hline & Brief MM & $S D$ & $n$ & Control & $S D$ & $n$ & Cohen's $d$ & $t$ & $p$ \\
\hline \multicolumn{10}{|l|}{ SAI } \\
\hline Baseline & 45.08 & $\begin{array}{l}14.3 \\
3\end{array}$ & 24 & 45.54 & 9.11 & 24 & 0.04 & -. 132 & .895 \\
\hline Post-SAI & 30.29 & 8.80 & 24 & 43.12 & 10.30 & 24 & 1.33 & -4.63 & $.000^{*}$ \\
\hline Difference & -14.79 & $\begin{array}{l}13.2 \\
0\end{array}$ & 24 & -2.41 & 12.71 & 24 & 0.95 & -3.307 & $.002^{*}$ \\
\hline $\begin{array}{l}\text { Cognitive } \\
\text { Performance }\end{array}$ & 11.13 & 3.10 & 23 & 11.70 & 3.30 & 24 & & -.617 & .540 \\
\hline
\end{tabular}


To determine whether sex and prior meditation experience had an effect on differences in SAI scores between groups, a series of two-way analyses of variance (ANOVAs) were conducted. Results indicated that there was no significant interaction between group and sex on SAI scores, $F$ $(1,44)=.091, p=.764, n_{p}^{2}=.002$.

The main effect for group was significant, $F(1$, $44)=9.06, p=.004, n_{p}^{2}=.171$, but the main effect for sex was not significant, $F(1,44)=3.71, p=.06$, $n_{p}^{2}=.078$. Further analyses revealed that the interaction between group and prior meditation experience was not significant, $F(1,44)=.740, p=$ $.39, n_{p}^{2}=.017$, nor was the main effect for prior meditation experience, $F(1,44)=.199, p=.658$, $n_{p}^{2}=.005$.

\section{Discussion}

We examined whether brief MM reduces SAI scores and promotes higher Cognitive Performance Test (CPT) scores when compared to a control group. As expected, there was a significant reduction in SAI scores in the brief MM group, such that state test-anxiety levels were lower in the brief MM group than the control group. Brief MM, however, did not promote any specific benefits for cognitive performance between groups.

Further analyses revealed sex and prior meditation experience had no effect on immediate state-test anxiety relief. Although females had greater state test-anxiety reduction than males at post-treatment, we should be cautious of interpreting these results as the sample's male to female ratio was significantly disproportionate, with 34 females and 14 males in the sample. Nonetheless, our findings with a college student sample are consistent with those of previous studies demonstrating the mood-enhancing effects of brief MM (Johnson et al., 2015).

The state test-anxiety reducing effects observed in the current study converge with prior studies examining the effectiveness of brief MM on mood. For instance, a three-session study found that 25-minutes of $\mathrm{MM}$ effectively reduced negative affect when compared to controls (Johnson et al., 2015). In the current study, a 20minute MM session produced comparable results in that SAI scores were significantly reduced at post-treatment when compared to the control group. Our findings are also consistent with those of Cruess et al. (2015) who found that 15-minutes of MM effectively reduced SAI scores when compared to controls.

Our findings further converge with those of another recent study that found that a 10 -minute MM session led to significant reductions in experimentally induced state-anxiety when compared to relaxation training (Ainsworth et al., 2015).

Decreased mind-wandering and enhanced emotional regulation may be especially relevant in explaining the present study's observed effect. It has been proposed that brief MM improves mood through its ability to develop emotional regulatory skills, inhibit mind-wandering, enhance attention and working memory capacity, and promote decentering (Ainsworth et al., 2015; see also Dickenson et al., 2013; Eddy et al., 2015; Feldman et a., 2010; Rahl et al., 2017).

Operating as a mechanism in MM, decentering refers to the act of noting and re-conceptualizing the way one views their thoughts, emotions, and sensations. As such, it involves perceiving mental and physical events with enhanced objectivity by changing or reperceiving one's relationship to their thoughts instead of attempting to alter the content of their thoughts (Safran \& Segal, 1990).

Previous work has suggested that brief MM may promote the integration of cognitive, affective, and sensory stimuli and the development of greater self- awareness and emotional regulation (Zeidan et al., 2014). In the current study, those who engaged in brief MM may have experienced a reduction in mind-wandering and a subsequent decrease in state test-anxiety.

The integration of cognitive, affective, and sensory stimuli may explain why those in the brief MM group had greater anxiety reduction when compared to the control group. Previous work suggests that the cultivation of present-moment awareness acts as a mechanism that facilitates mood improvement. Specifically, brief MM has been evidenced to target worry cognitions and attentional challenges associated with negative mood through the reappraisal and reconsolidation of emotion (Ainsworth et al., 2015), and the activation of attention-related neural correlates (Dickenson et al., 2013).

In the present study, we speculate that a reduction in SAI scores may be attributed to participants' ability to nonjudgmentally notice and reappraise their feelings, thoughts, and sensations 
with greater objectivity. The brief MM used in the current study focused on noting current mental and physical events with less intensity which may explain the reduction in SAI scores. More specifically, an ability to decenter may have contributed to a reduction in state-anxiety in the brief MM group.

Decentering may be particularly relevant in explaining how brief MM may facilitate mood enhancement, attenuate worry cognitions, and decrease ruminative thinking (Eddy et al., 2015; Feldman et al., 2010). An individual's ability to reperceive their thought processes has been argued to foster the ability to respond to internal and external events with greater emotional regulation, greater objectivity, and less intensity (Eddy et al., 2015; Erisman \& Roemer, 2010; Roemer et al., 2015).

Specifically, an enhanced state of mindfulness, or present-moment awareness, may be especially relevant in explaining the effectiveness of brief MM on mood. In a recent study, a single five-minute MM audio recording significantly increased levels of state mindfulness in the brief MM group, but not in the control group (Mahmood et al., 2016).

In the current study, it is possible that a reduction in state test-anxiety is the result of the brief MM audio recording explicitly instructing participants to focus and accept their current emotions and thoughts. We postulate that the act of focusing on the present-moment in relation to current mental and physical events resulted in immediate state test-anxiety relief in our college student sample.

The immediate, short-term benefits of brief MM may have clinical implications. First, extensive MM training regimens typically require a great deal of time and monetary commitment (Santorelli, 2014). For students with limited time and money, brief MM is not only cost-effective, but it does not require a copious amount of time. As such, students will not be burdened by a counselling office waitlist and instead can engage in brief MM when it fits their schedule (Shearer et al., 2016).

Additionally, brief MM can be accessed more conveniently online, such as smartphone applications. The accessibility and immediacy that brief MM offers may be particularly attractive to a college-student populace. As testing continues to dominate post-secondary education, perhaps briefer formats of MM may be just as ever-present.
Although state test-anxiety decreased at posttreatment, brief MM did not lead to higher CPT scores in our sample. Previous work has suggested that brief MM can improve cognitive performance through the promotion of executive attention and attentional focus (Watier \& Dubois, 2016).

For instance, a recent study found that a single 10-minute MM session improved participants' ability to focus on the Stroop Test (an experiment wherein a series of coloured words are displayed, and a respondent is required to say the colour of the word and not the word itself) when compared to an attention exercise control condition and an arithmetic exercise control condition. In the current study, 20-minutes of MM may have been ineffective at improving cognitive performance in a college student sample.

A lack of difference between groups on cognitive performance may be explained by recent evidence suggesting that brief MM may only result in immediate, short-term cognitive benefits (Calma-Birling \& Gurung, 2017).

Examining the effects of brief $\mathrm{MM}$ on test performance in an undergraduate sample, a sevenweek study found that five-minute inductions of MM boosted quiz grades and short-term knowledge retention, but failed to impact longterm test scores (Calma-Birling \& Gurung, 2017). As such, smaller doses of MM may only give rise to immediate, short-term cognitive performance effects (Calma-Birling \& Gurung, 2017).

These results were consistent with those of Hafenbrack and Vohs (2018) who found that a series of six to 15-minute MM sessions were unable to impact task performance on word puzzles relative to control conditions. Enhanced cognitive performance may require lengthier mindfulness training (eight to 12-weeks), rather than brief doses of MM.

In the current study, brief MM may have failed to yield immediate cognitive performance improvements due to an insufficient duration of MM. Direct cognitive performance improvements may require a greater amount of time, commitment, and practice. Additionally, brief MM may only lead to short-term anxiety relief that may dissipate in the face of an anxiety inducing situation. Longer term MM interventions may be necessary to promote the habits required to maintain mindfulness amidst stressful events (i.e., 18 weeks; Ching et al., 2015). 
Lack of improvement in cognitive performance in the current study may also be attributed to a failure to promote meta-awareness (Epel et al., 2009; Franklin et al., 2017; Farb et al., 2007). Meta-awareness is often conceptualized as the ability to reflect on current internal and external experiences (Smallwood et al., 2007).

There is increasing evidence that MM is largely connected to brain networks that are responsible for executive functioning, meta-awareness, and mind-wandering (Epel et al., 2009; Farb et al., 2007; Franklin et al., 2017). Mind-wandering, in particular, has been shown to impair cognitive processes and executive functioning through its ability to inhibit goal-directed attention (Franklin et al., 2017; Smallwood et al., 2007).

When the mind begins to wander, taskirrelevant thoughts interfere with cognitive functioning which can result in distraction from the task itself (Smallwood \& Schooler, 2006). When distraction occurs, a disruption in one's metaawareness ensues (Smallwood \& Schooler, 2006). Since brief MM is premised on noticing and reappraising task-irrelevant thoughts, feelings, and emotions, the mind often wanders less through the promotion of meta-awareness (Franklin et al., 2017).

In the current study, it is possible that the MM intervention was ineffective at mitigating the production of task-irrelevant thoughts from interfering with the present-moment, thereby failing to promote meta-awareness. A lack of effect on cognitive performance may have been exasperated by prominent limitations in the current study.

\section{Limitations and Future Research}

The fundamental aspects of the current study presented several limitations. First, the questions used in the Anxiety Induction Test (AIT)were arbitrarily chosen by the researcher from a preexisting, non-standardized measure. Although the test underwent a mock trial run and was deemed anxiety-producing (by participants distinct from those in the actual experiment), there is no way of knowing whether the AIT actually produced a state-level test-anxiety response. Future research would benefit from using a standardized testing measure that has been empirically evidenced to elicit state-test anxiety.

Second, it is possible that the highly contrived nature of the current study failed to mirror the realities of college examinations. The standardized nature of the CPT may have counteracted the effects of brief MM.

Last, the CPT test used in the current study was arbitrarily designed from a reliable intelligence examination. No formal validation process was employed to ensure that the questions chosen were measuring cognitive performance. The measure used to test cognitive performance may be relevant in explaining a lack of difference between groups in the current study. Consequent to an arbitrary adaption process, the measure may have failed to mirror the realities of a lecture-driven college examination as seen in campus classrooms.

A lack of difference between groups on cognitive performance may be due to the differences between assessing knowledge of information on a test of general intelligence and a test assessing recently learned information. Previous work has examined test performance in the context of lecture knowledge retention (e.g., Calma-Birling \& Gurung, 2017) and prior examination preparation (i.e., GRE examination preparation), but not in the context of recalling information on a test of general intelligence (Mrazek et al., 2013). As such, there may be differences between tests assessing previously learned material and tests assessing general intelligence.

Recent findings have suggested that mental training programs, such as brief $\mathrm{MM}$, do not have the ability to improve cognitive performance on standardized measures of intelligence (MelbyLervåg et al., 2016). This suggests that performance on a standardized measure may be impervious to brief MM. In the current study, there was no previous material from which subjects could study prior to taking the CPT. Given that this measure was also adapted from a standardized measure of intelligence, it is possible that the brief MM session was ineffective enhancing participants' ability to focus and recall general information on the test. Formal validation of the cognitive performance measure is recommended for future MM research.

It is also recommended that future research utilize a quasi-experimental design to examine the effects of brief MM on lecture-based examinations during scheduled college class times. Using this type of design may also eliminate the possibility of participants responding in a manner that confirms their beliefs of the research study. 
The duration of MM used in the present study may also explain a lack of difference in CPT scores. Previous work has typically used lengthier training programs ranging from two- weeks (i.e., Mrazek et al., 2013) to seven-weeks (i.e., Calma-Birling \& Gurung, 2017) to 14 or more weeks (Bellinger et al., 2015), whereas, the present study utilized a 20minute MM session. A lengthier MM session may be necessary to replicate the results observed in previous studies. It is recommended that future research examine the immediate effectiveness of both single-session MM inductions and briefer MM training programs to determine the appropriate amount of time, commitment, and training needed to achieve enhanced cognitive performance in college age populations.

Additionally, the present study lacked the use of an in-person mindfulness facilitator, and instead used an audio-recording. Gaining a better understanding of the physical-virtual distinction of brief MM would be a beneficial venture for further research in this area. Nonetheless, it is important to note that even with an auditory experience, brief MM can effectively reduce state-anxiety in an undergraduate sample. A more thorough analysis of sex differences is also recommended for future research to determine whether brief MM results in greater state test-anxiety reduction for females or males. Equal proportions of males and females in each group would accomplish this analysis.

Overall, findings of the present study suggest that brief MM may produce immediate state-level test-anxiety relief in college age populations. Although promising, we do not contend that brief $\mathrm{MM}$ is as effective as lengthier mindfulness-based interventions but intend to show that it has the potential to be an effective state-anxiety reduction technique for students in stressful examination settings.

As opposed to a more general, reoccurring test-anxiety response, our research supports the contention that briefer formats of MM may prove more beneficial for short-term, present-moment test-anxiety. Results further highlight the significance in examining the effects of brief MM on cognitive functioning and test performance in student populations. Exploring the cognitive benefits of brief MM may be advantageous to not only the advancement of test-anxiety research, but students' mental health and future academic achievement.

\section{References}

Abolghasemi, A., Golpour, R., Narimani, M., \& Ghambari, H. (2009). Studying the relationship between negative metacognitive beliefs and academic success in test anxious students. Educational Studies and Psychology, 10(3), 5-20.

Ainsworth, B., Marshall, J. E., Meron, D., Baldwin, D. S., Chadwick, P., Munafò, M. R., \& Garner, M. (2015). Evaluating psychological interventions in a novel experimental human model of anxiety. Journal of Psychiatric Research, 63, 117122.

http://dx.doi.org/10.1016/i.jpsychires.20 $\underline{15.02 .001}$

Banks, J. B., Welhaf, M. S., \& Srour, A. (2015). The protective effects of brief mindfulness meditation training. Consciousness and Cognition: An International Journal, 33, 277-285.

http://dx.doi.org/10.1016/j.concog.2015. $\underline{01.016}$

Beilock, S. L., \& Ramirez, G. (2011). On the interplay of emotion and cognitive control: Implications for enhancing academic achievement. Psychology of Learning and Motivation, 55, 137-169. https://doi.org/10.1016/B978-0-12387691-1.00005-3

Bellinger, D. B., DeCaro, M. S., \& Ralston, P. A. (2015). Mindfulness, anxiety, and highstakes mathematics performance in the laboratory and classroom. Consciousness and Cognition, 37, 123-132. https://doi.org/10.1016/j.concog.2015.0 $\underline{9.001}$

Brady, S. T., Hard, B. M., \& Gross, J. J. (2018). Reappraising test anxiety increases cognitive performance of first-year college students. Journal of Educational Psychology, 110(3), 395-406. https://doi.org/10.1037/edu0000219.su pp

Broderick, P. C. (2005). Mindfulness and coping with dysphoric mood: Contrasts with rumination and distraction. Cognitive Therapy and Research, 29, 501-510. https://doi.org/10.1007/s10608-005$\underline{3888-0}$ 
Calma-Birling, D., \& Gurung, R. R. (2017). Does a brief mindfulness intervention impact quiz performance? Psychology Learning and Teaching, 16(3), 323-335. https://doi.org/10.1177/147572571771 $\underline{2785}$

Cavanagh, K., Strauss, C., Cicconi, F., Griffiths, N., Wyper, A., \& Jones, F. (2013). A randomized controlled trial of a brief online mindfulness-based intervention. Behaviour Research \& Therapy, 51(9), 573578.

https://doi.org/10.1016/j.brat.2013.06.0 $\underline{03}$

Chin, E. C., Williams, M. W., Taylor, J. E., \& Harvey, S. T. (2017). The influence of negative affect on test anxiety and academic performance: An examination of the tripartite model of emotions. Learning and Individual Differences, 54, 1-8. https://doi.org/10.1016/j.lindif.2017.01. $\underline{002}$

Ching, H.-H., Koo, M., Tsai, T.-H., \& Chen, C.-Y. (2015). Effects of a mindfulness meditation course on learning and cognitive performance among university students in Taiwan. Evidence-Based Complementary and Alternative Medicine, 2015, 1-7. http://dx.doi.org/10.1155/2015/254358

Cho, H., Ryu, S., Noh, J., \& Lee, J. (2016). The effectiveness of daily mindful breathing practices on test anxiety of students. PLoS ONE, 11(10), 1-10. https://doi.org/10.1371/journal.pone.01 $\underline{64822}$

Cruess, D. G., Finitsis, D. J., Smith, A.-L., Goshe, B. M., Burnham, K., Burbridge, C., \& Oleary, K. (2015). Brief stress management reduces acute distress and buffers physiological response to a social stress test. International Journal of Stress Management, 22(3), 270-286. http://doi.org/10.1037/a0039130

Dickenson, J., Berkman, E. T., Arch, J., \& Lieberman, M. D. (2013). Neural correlates of focused attention during a brief mindfulness induction. Social Cognitive and Affective Neuroscience, 8, 4047. http://dx.doi.org/10.1093/scan/nss030
Dundas, I., Thorsheim, T., Hjeltnes, A., \& Binder, P. E. (2016). Mindfulness based stress reduction for academic evaluation anxiety: A naturalistic longitudinal study. Journal of College Student Psychotherapy, 30(2), 114-131.

https://doi.org/10.1080/87568225.2016. 1140988

Dvořáková, K., Kishida, M., Li, J., Elavsky, S., Broderick, P. C., Agrusti, M. R., \& Greenberg, M. T. (2017). Promoting healthy transition to college through mindfulness training with first-year college students: Pilot randomized controlled trial. Journal of American College Health, 65(4), 259-267. https://doi.org/10.1080/07448481.2017. $\underline{1278605}$

Eddy, M. D., Brunyé, T. T., Tower-Richardi, S., Mahoney, C. R., \& Taylor, H. A. (2015). The effect of a brief mindfulness induction on processing of emotional images: An ERP study. Frontiers in Psychology, 6(1391). 112.

https://doi.org/10.3389/fpsyg.2015.0139 $\underline{1}$

Epel, E., Daubenmier, J., Moskowitz, J. T., Folkman, S., \& Blackburn, E. (2009). Can meditation slow rate of cellular aging?

Cognitive stress, mindfulness, and telomeres. Annals of the New York Academy of Sciences, 1172, 34-53. https://doi.org/10.1111/j.17496632.2009.04414.x

Erisman, S., \& Roemer, L. (2010). A preliminary investigation of the effects of experimentally induced mindfulness on emotional responding to film clips. Emotions, 10(1), 72-82. https://dx.doi.org/10.1037\%2Fa0017162

Farb, N. A., Segal, Z. V., Mayberg, H., Bean, J., McKeon, D., Fatima, Z., et al (2007). Attending to the present: Mindfulness meditation reveals distinct neural modes of self-reference. Social Cognitive Affective Neuroscience, 2(4), 313-322. https://doi.org/10.1093/scan/nsm030

Feldman, G., Greeson, J., \& Senville, J. (2010). Differential effects of mindful breathing, progressive muscle relaxation, and loving kindness meditation on decentering and negative reactions to repetitive thoughts. 
Behavior Research and Therapy, 48, 10021011. https://doi.org/10.1016/j.brat.2010.06.0 $\underline{06}$

Franklin, M. S., Mrazek, M. D., Anderson, C. L., Johnston, C., Smallwood, J., Kingstone, A., \& Schooler, J. W. (2017). Tracking distraction: The relationship between mind-wandering, meta-awareness, and ADHD symptomatology. Journal of Attention Disorders, 21(6), 475-486. https://doi.org/10.1177/108705471454 $\underline{3494}$

Galante, J., Dufour, G., Vainre, M., Wagner, A. P., Stochl, J., Benton, A., ... \& Jones, P. B. (2018). A mindfulness-based intervention to increase resilience to stress in university students (the Mindful Student Study): A pragmatic randomised controlled trial. The Lancet Public Health, 3(2), 72-81.

https://doi.org/10.1016/S24682667(17)30231-1

Gerwing, T. G., Rash, J. A., Gerwing, A. M. A., Bramble, B., \& Landine, J. (2015). Perceptions and Incidence of Test Anxiety. Canadian Journal for the Scholarship of Teaching \& Learning, 6(3), 1. http://dx.doi.org/10.5206/cjsotlrcacea.2015.3.3

Hafenbrack, A. C., \& Vohs, K. D. (2018). Mindfulness meditation impairs task motivation but not performance. Organizational Behavior and Human Decision Processes, 147, 1-15. https://doi.org/10.1016/j.obhdp.2018.05. $\underline{001}$

How to Origami. (2014, December 26). How to Fold an Origami F-16 Paper Plane [Video file]. Youtube. https://www.youtube.com/watch?v=4nkj b1LsD64

Jayawardene, W., Erbe, R., Lohrmann, D., \& Torabi, M. (2017). Use of treatment and counseling services and mind-body techniques by students with emotional and behavioral difficulties. Journal of School Health, 87(2), 133-141. https://doi.org/10.1111/josh.12475

Johnson, S., Gur, R. M., David, Z., \& Currier, E. (2015). One-session mindfulness meditation: A randomized controlled study of effects on cognition and mood. Mindfulness, 6(1), 88-98. https://doi.org/10.1007/s12671-013$\underline{0234-6}$

Kabat-Zinn, J. (1982). An outpatient program in behavioral medicine for chronic pain patients based on the practice of mindfulness meditation: Theoretical considerations and preliminary results. General Hospital Psychiatry, 4, 33-47. https://doi.org/10.1016/01638343(82)90026-3

Kabat-Zinn, J. (2003), Mindfulness-Based Interventions in Context: Past, Present, and Future. Clinical Psychology: Science and Practice, 10: 144-156. https://doi.org/10.1093/clipsy.bpg016

Kabat-Zinn, J. (2014). Sitting Meditations-20 minutes. [Recorded by Jon Kabat Zinn]. On [MP3 file]. Louisville, Colorado: Soundstrue, Inc.

Lothes, J. E., \& Mochrie, K. (2017). The "what" and "hows" of mindfulness: Using DBT's mindfulness skills to reduce test anxiety. Building Healthy Academic Communities Journal, 1(2), 10-20. https://doi.org/10.18061/bhac.v1i2.6029

Mahmood, L., Hopthrow, T., \& de Moura, G. R. (2016). A moment of mindfulness: Computer-mediated mindfulness practice increases state mindfulness. PloS One, 11(4). https://doi.org/10.1371/journal.pone.01 $\underline{53923}$

Morrison, A. B., Goolsarran, M., Rogers, S. L., \& Jha, A. P. (2014). Taming a wandering attention: Short-form mindfulness training in student cohorts. Frontiers in Human Neuroscience, 7, 897. https://doi.org/10.3389/fnhum.2013.008 $\underline{97}$

Melby-Lervåg, M., Redick, T. S., \& Hulme, C. (2016). Working memory training does not improve performance on measures of intelligence or other measures of "far transfer" evidence from a meta-analytic review. Perspectives on Psychological Science, 11(4), 512-534. https://doi.org/10.1177/174569161663 $\underline{5612}$

Mrazek, M. D., Franklin, M. S., Phillips, D. T., Baird, B., \& Schooler, J. W. (2013). 
Mindfulness training improves working memory capacity and GRE performance while reducing mind wandering. Psychological Science, 24(5), 776-781. https://doi.org/10.1177/095679761245 $\underline{9659}$

Nausheen, M., Richardson, P. W., \& Alvi, E. (2019). Patterns of gender and disciplinary disparities among postgraduate students' motivational beliefs: A multivariate analysis. Bulletin of Education and Research, 41(1), 203-220. https://pdfs.semanticscholar.org/617c/d 27281986f7be3588423e1ae30ab98c295b 4.pdf?_ga $=2.40848098 .1724629388 .1588$ 807657-44417998.1569039541

Otis, A. S. (1920). Otis General Intelligence Examination [Database record]. PsycTESTS. http://dx.doi.org/10.1037/t00650-000

Rahl, H. A., Lindsay, E. K., Pacilio, L. E., Brown, K. W., \& Creswell, J. D. (2017). Brief mindfulness meditation training reduces mind wandering: The critical role of acceptance. Emotion, 17, $224-230$. http://dx.doi.org/10.1037/emo0000250

Sapp, M. (2014). Test anxiety: Applied research, assessment, and treatment interventions ( $3^{\text {rd }}$ ed.). University Press of America.

Santorelli, S. (2014). Mindfulness-based stress reduction (MBSR): Standards of practice. The Center for Mindfulness in Medicine, Health Care, and Society. https://www.umassmed.edu/contentasse ts/24cd221488584125835e2eddce7dbb8 9/mbsr_standards_of_practice_2014.pdf

Safran, J.D., \& Segal, Z.V. (1990). Interpersonal process in cognitive therapy. Rowan and Littlefield Publishers.

Shearer, A., Hunt, M., Chowdhury, M., \& Nicol, L. (2016). Effects of a brief mindfulness meditation intervention on student stress and heart rate variability. International Journal of Stress Management, 23(2), 232254.

http://dx.doi.org/10.1037/a0039814

Smallwood, J., McSpadden, M., \& Schooler, J. W. (2007). The lights are on but no one's home: Meta-awareness and the decoupling of attention when the mind wanders. Psychonomic Bulletin and
Review, 14(3), 527-533. http://dx.doi.org/10.3758/BF03194102

Smallwood, J., \& Schooler, J. W. (2006). The restless mind. Psychological Bulletin, 132(6), 946-958. https://doi.org/10.1037/00332909.132.6.946

Spielberger, C. D. (1966). Theory and research on anxiety. Anxiety and behavior. Academic Press.

Spielberger, C. D. (1972). Anxiety: current trends in theory and research. Academic Press.

Spielberger, C. D., Anton, W. D., \& Bedell, J. (1976). The nature and treatment of test anxiety. In M. Zuckerman \& C. D. Spielberger (Eds.). Emotions and anxiety: New concepts, methods, and applications (1st ed., pp. 317-344). Psychology Press.

Spielberger, C. D., Gorsuch, R. L., \& Lushene, R. E. (1983). The State-trait anxiety inventoryForm Y1 (STAI-Y1). Technical Manual. Mind Garden.

Spielberger, C. D., \& Vopp, P.R. (1995). Test anxiety: Theory, assessment, and treatment. Taylor and Francis.

Stoker, J. \& Perkin, R. (2014). Advances in test anxiety research. Anxiety, Stress, \& Coping, 17(3), 205-211.

https://doi.org/10.1080/106158041233 $\underline{1303225}$

Roemer, L., Williston, S. K., \& Rollins, L. G. (2015). Mindfulness and emotion regulation. Current Opinion in Psychology, 3, 52-57. https://doi.org/10.1016/j.copsyc.2015.02 .006

Taren, A. A., Gianaros, P. J., Greco, C. M., Lindsay, E. K., Fairgrieve, A., Brown, K. W., ... \& Creswell, J. D. (2017). Mindfulness meditation training and executive control network resting state functional connectivity: A randomized controlled trial. Psychosomatic Medicine, 79(6), 674. https://doi.org/10.1097/PSY.000000000 0000466

The Impossible Quiz. (2018). The Impossible Quiz - Nobody gets all the answers to these trick questions [Database record]. http://www.impossible-quiz.com/

Thomas, C. L., Cassady, J. C., \& Heller, M. L. (2017). The influence of emotional intelligence, cognitive test anxiety, and 
coping strategies on undergraduate academic performance. Learning and Individual Differences, 55, 40-48. https://doi.org/10.1016/j.lindif.2017.03. $\underline{001}$

Trammell, J. P. (2017). The effect of therapy dogs on exam stress and memory. Anthrozoos, 30(4), 607-621. https://doi.org/10.1080/08927936.2017. 1370244

von der Embse, N., Barterian, J., \& Segool, N. (2013). Test anxiety interventions for children and adolescents: A systematic review of treatment studies from 20002010. Psychology in the Schools, 50(1), 5771. https://doi.org/10.1002/pits.21660

Watier, N., \& Dubois, M. (2016). The effects of a brief mindfulness exercise on executive attention and recognition memory. Mindfulness, 7(3), 745-753. https://doi.org/10.1007/s12671-0160514-Z

Wells, A., \& Roussis, P. (2014). Refraining from intrusive thoughts is strategy dependent: A comment on Sugiura, et al. and a preliminary informal test of detached mindfulness, acceptance, and other strategies. Psychological Reports, 115(2), 541. http://dx.doi.org/10.2466/02.PR0.115c2 $\underline{1 \mathrm{z} 9}$

Xu, M., Purdon, C., Seli, P., \& Smilek, D. (2017). Mindfulness and mind wandering: The protective effects of brief meditation in anxious individuals. Consciousness and cognition, 51, 157-165. https://doi.org/10.1016/j.concog.2017.0 $\underline{3.009}$

Zeidan, F., Gordon, N. S., \& Goolkasian, P. (2009). The effects of brief meditation training on experimentally induced pain perception. Journal of Pain, 10, 1016-1019. https://doi.org/10.1016/i.jpain.2009.07.0 $\underline{15}$

Zeidan, F., Johnson, S. K., Diamond, B. J., David, Z., \& Goolkasian, P. (2010a). Mindfulness meditation improves cognition: Evidence of brief mental training. Consciousness and Cognition, 9(2), 597-605. http://dx.doi.org/10.1016/j.concog.2010. $\underline{03.014}$
Zeidan, F., Johnson, S. K., Gordon, N. S., \& Goolkasian, P. (2010b). The effects of brief and sham mindfulness meditation on mood and cardiovascular variables. The Journal of Alternative and Complementary Medicine, 16, 867-873. http://dx.doi.org/10.1089/acm.2009.032 1

Zeidan, F., Martucci, K. T., Kraft, R. A., McHaffie, J. G., \& Coghill, R. C. (2014). Neural correlates of mindfulness meditationrelated anxiety relief. Social Cognitive and Affective Neuroscience, 9(6), 751-759. https://doi.org/10.1093/scan/nst041

Zeidner, M. (1998). Test Anxiety: The State of the Art. Plenum Press. 